\title{
Imagery, rehearsal, and the compatibility of input-output tasks
}

\author{
M. J. PETERSON, JACK E. THOMAS, and HOLLY JOHNSON \\ Indiana University, Bloomington, Indiana 47401
}

\begin{abstract}
Two experiments tested the proposition that recall in an imagery task would be facilitated when the processes instigated during original input and during a rehearsal interval were similar to (compatible with) processes assumed to be initiated by two retrieval formats. In both experiments the subjects listened to tape-recorded messages which described the placements of numbers in an imaginary mental matrix. Experiment 1 used four modes of presenting the original information about the placement of the numbers. Two of the input modes were expected to foster the use of imagery: listening to the tape-recorded messages $(\mathrm{L})$, and listening while shadowing (vocalizing) the messages (LV). The other modes of presentation were expected to encourage verbalized rather than imaginal encoding: listening plus silent reading (LR), and listening plus reading aloud (LRV). Two types of recall tasks were used, one which emphasized imaginal coding (matrix recall) and one which emphasized verbal coding (direction recall). Recall was highest when the input and output tasks were assumed to involve similar types of processing. Thus, Groups L and LV showed higher recall than Groups LR and LRV on the matrix task, but the converse was true for the direction task. In addition, rotation of the information yielded different characteristics for the input-output conditions. Experiment 2 introduced rehearsal instructions. Visualizing rehearsal was more beneficial to matrix recall than verbalizing rehearsal or no explicit rehearsal instructions, and verbalizing rehearsal aided direction recall more than visualizing rehearsal. Various models were discussed.
\end{abstract}

The two experiments presented in this paper studied the relationships between the characteristics of the original input material, assumed encoding processes, and final output in an imagery task. The basic task instructed students to enter numbers in an imaginary matrix in a manner described by tape-recorded messages. To study the relationships between input and output recall in this task, the basic paradigm was modified for some groups by (1) increasing the number of redundant modes of input (e.g., listening to the messages, reading the messages while listening to them), (2) assessing output recall in two different ways (entering numbers in a printed matrix or recalling the directions of placement of the numbers), (3) varying the extent of mental rotation required before recall, and (4) instructing the subjects to employ different types of rehearsal strategies (visualizing or verbalizing the information during a rehearsal interval).

This type of task is ideal for studying visual imagery when the basic information about what is to be imaged is presented in a form that requires transformation into an image and when different recall methods are used. In our opinion, one promising theoretical approach to this type of visual imagery task is a model which emphasizes processing, such as a modified levels of processing view. Our conceptualization of such a framework is

The authors wish to thank Jerry Sinsabaugh for his invaluable assistance. Requests for reprints should be sent to $M$. J. Peterson, Department of Psychology, Indiana University, Bloomington, Indiana 47401. that the verbal messages initially undergo a series of structural, phonemic, and semantic processing which produce a distinctive set of by-products. These byproducts, the "meanings" of the messages, constitute "input" for the additional processing involved in "imaginal representation." Hence, it was assumed that the auditory messages would be processed in a manner customarily utilized for auditory input and the "meaning" or some resultant of the preliminary processing would then be subjected to additional processing to construct a representation, the image.

This conceptualization is a straightforward extension of a levels of processing notion to include the assumption that imaginal processing involves additional elaboration. The approach predicts that different types of input (e.g., listening and reading) which convey the same "meaning" will elicit differentiable but similar types of processing. This prediction was tested in the experiments described herein.

Further, we assumed that the effectiveness of recall is directly related to the extent to which the recall test reinstitutes or reconstitutes processing similar to the original encoding. For example, in the task described above, entering appropriate numbers into a printed matrix would reinstitute processes similar to (compatible with) those involved in the original imagery task. A recall test whose characteristics are compatible with the image should yield better performance than a recall test whose characteristics differ from the hypothetical imaginal representation. This view is similar to the model 
proposed by Lockhart, Craik, and Jacoby (1976). They extended the Craik and Lockhart (1972) position to encompass retrieval processes. Their proposal is that there are two kinds of retrieval processes: scanning and reconstruction. For purposes of this paper, we focus on the reconstructive aspects of retrieval.

These ideas are developed below. At this point, it is important to note the similarities in prediction between the levels of processing approach and more associative models such as those espoused by Anderson (1976) and Paivio (see Paivio, 1976, for a recent review). Both models allow for the encoding of distinctive characteristics of the input and for recall performance based on the compatibility or similarity of the recall task with the encoding processes.

Increasing the number of redundant modes of input could have various effects. Suppose that the processing customarily instituted by the various sources of input retain or contain some aspects distinctive to the sources of input, in addition to the postulated resultants of the processing, the meanings of the messages. Then when subjects read the messages while listening to them, both visual and auditory processes would be instigated although the basic "meaning" would be the same regardless of the source of input. The distinctive characteristics of the processings (1) should aid retrieval when the recall task is devised to cue some of the distinctive aspects of the input tasks, and (2) would be enhanced by the use of various recall strategies only when the rehearsal processes are compatible with or similar to the processes in. volved in input and output.

Experiment 1 involved four input conditions (groups). All four groups listened to the messages. One of the groups (LR) read the messages silently as they heard them; another group (LV) shadowed the messages as they heard them; a third group (LRV) read the messages aloud as they listened to the messages. The fourth group (L) listened only. Thus, Group L had a single source of input (auditory). Groups LR and LV had at least two sources of input (auditory plus visual or auditory plus articulatory), and Group LRV had at least three sources (auditory, visual, articulatory). Half of the subjects received a "matrix" recall test; the other half of the subjects received a "direction" recall test. For matrix recall the subject was to enter, accurately, the appropriate numbers in the cells of a 4 by 4 blank matrix. This type of test was assumed to be particularly compatible with an imaginal processing (visualization) of the input information. For direction recall the subject's task was to enter the correct direction of movement before each of the numbers. Direction recall was assumed to rely heavily on specific reading cues because portions of the test were highly similar to the typewritten messages read by some groups of subjects.

The theoretical framework described above predicted interactions between the processes instigated by the input modes and the recall tests. Specifically, it was expected that listening and, perhaps, listening plus vocalizing, would encourage the construction of a mental image and that retrieval of the contents of the matrix in this situation would be maximized by the matrix type of recall test. Performance on the direction type of recall test should be higher when the subjects read the materials, either silently or aloud.

In contrast to the interactions predicted above, a number of other models predict that recall, regardless of the type of test, would be monotonically related to the number of input modes. One position was that, if the multiple processing of the input modes operated to replicate the meanings of the messages, recall should increase directly with increases in the number of input modes. This possibility was not entertained seriously because Brooks (1967) reported lower retention of the contents of mental matrices when subjects read the messages as they listened to the descriptions compared to performance when subjects simply listened to the messages. Brooks attributed the difference to interference with visual processing created by simultaneously reading and imagining the mental matrix, although he also considered the possibility of an overloading of the system as a factor. When applied to the conditions of Experiment 1, the position that the processes invoked by reading would interfere with the construction of the mental image and the overload possibility both predicted an inverse relationship between the number of input modes of the type used in Experiment 1 and recall.

Posner and Snyder's (1975) position that the conscious focusing of attention, an intentional process, upon single sources of input would produce facilitation, while attending to multiple sources would generate interference, also predicted an inverse relationship between the input sources and recall. Their model suggested that the best performance would be shown by the group which experienced minimal input interference, the group that listened only. Poorest recall would be found for the group with the largest number of potentially interfering inputs, the group that listened, read, and vocalized.

Thus, there were a number of predictions made for the first experiment, one of which advanced the notion of an interaction between the type of recall and the conditions of input. The first experiment tested these predictions by presenting the information under four conditions of input and using both matrix and directional recall to test retention. The two types of recall tests assessed the same basic information and required the same number of responses from the subjects, so the tests were assumed to be approximately equal in difficulty.

In addition to the variables already cited, Experiment 1 introduced a rotation requirement to study the ability of subjects to perform mental manipulations of their images. The subjects were asked to rotate their imaginary matrices through $0,90,180$, or $270 \mathrm{deg}$ before recall. 
The rotational variable permitted a comparison of some of the dynamics of recall in the two retrieval situations. Wickelgren (1975) has argued that different types of memory traces should be posited only if the evidence indicates dynamically different phenomena. While some data suggested that verbal materials were recalled better at $180-\mathrm{deg}$ rotations than at other rotations (e.g., Kolers, 1968; Kolers \& Perkins, 1969, 1975; Smith, Cambria, \& Steffan, 1964), other research has exhibited a relatively smooth decline in recall from the original position when nonverbal materials were rotated (e.g., Cooper, 1975; Cooper \& Shepard, 1973). Consequently, the rotational variable was included to assess possible interactions with the other variables.

\section{EXPERIMENT 1}

\section{Method}

Material. Five practice messages and 16 regular experimental messages were constructed. The practice messages instructed the students to place the numbers $1-8$ in a 4 by 4 matrix. The first sentence of each eight-sentence message was the same: "In the starting square put a 1." The other seven sentences of the messages directed the students to place the numbers in consecutive numerical order in an adjacent square, by moving in one of four possible directions: up, down, right, or left. The numbers were contained within the boundaries of the matrix and only one digit was assigned to a single square. The students always began with number 1 in the square that marked the intersection of the second row and the second column. Ten seconds after the termination of the messages, the student was told to "Rotate $0(90,180,270)$ degrees." The students were instructed to rotate the matrices in a clockwise direction. Thirty seconds (20 sec for practice messages) after the rotation instruction, the student was told to "Write your responses." All messages and the instructions were taped for presentation on a Wollensak tape recorder. The students were instructed to record the final outcome of any rotations on specially prepared response sheets.

Four tapes were prepared to counterbalance the rotation for each message. Thus, each message was tested under each rotation, and each rotation was represented once in the last four practice messages and four times in the regular messages. The first practice message was shown under each rotation to demonstrate the procedure to the students.

Each message also was typed on a $3 \times 5$ in. $(7.6 \times 12.7 \mathrm{~cm})$ index card, and decks of cards were collated to correspond to the messages on the tapes. No rotation directions were typed on the cards.

The response sheets for matrix recall were sheets of graph paper. Students were told to enter the appropriate numbers into the correct cells. The response sheets for direction recall had blanks before each number. The students were to record letters identifying the correct direction in each blank. For example, the direction recall sheets contained " $\ldots 2, \ldots \ldots$, " and so on. If the directions in the messages indicated that the " 2 " should be placed in the square to the left of the starting square, the correct entry before " 2 " would be " $L$ " for "left."

Design. All students listened to the messages played from a Wollensak tape recorder through earphones. One group listened only (L). A second group silently read the messages from the cards as they listened to the messages over the earphones (LR). A third group vocalized aloud the messages as they listened and read them (LRV). The LR and LRV groups were instructed to read the messages in time to the auditory input. This technique was used to reduce individual differences in reading speed and consequent opportunities for rehearsal. The four levels of rota- tion were within-subjects effects. Half of each group was tested for matrix recall and half for direction recall.

Procedure. The experimenter used the first practice message to demonstrate the procedure including the possible rotations. Then the students received the appropriate instructions for processing the messages and were shown the 4 practice and the 16 regular messages. For matrix recall they recalled each message by writing the appropriate numbers into the cells of the blank matrix. For direction recall they indicated the direction of movement by writing one of four letters before the numbers used in the messages. The four letters were $U, D, R$, and $L$ to indicate "up," "down," "right," and "left," respectively.

Subjects. The subjects were 128 women and men enrolled in introductory psychology courses at Indiana University. They participated as one way to satisfy requirements of the courses. The students were tested in squads of up to four people. The squads were randomly assigned to 16 replications of the eightcell block (four groups and two types of test formats). Additional students were tested to fill incomplete squads, so that each group contained 16 people tested for matrix recall and 16 people tested for direction recall. The four tapes were used equally often with each group.

\section{Results}

Recall was considered correct only if all entries were correct for the final outcome of each rotation of each matrix. The recall scores were analyzed for the two recall formats by the four groups taking the rotations into account and separating the 16 regular trials into two halves. Figure 1 presents the proportionate recall for the above variables, save the separation into two halves.

There were a number of predictions to be assessed. If recall was directly related to the numbers of input modes, the ordering of the groups from highest to lowest recall would be $L R V>(L R, L V)>L$. However, if the multiple sources of input produced interference, the order of the groups would be reversed: $L>(L V, L R)>$ LRV. The hypothesis of interference between the visual processes associated with reading and the formation of an image yielded an ordering with the groups that did not read the messages recalling more than the groups that did read the messages: $(L, L V)>(L R, L R V)$. These

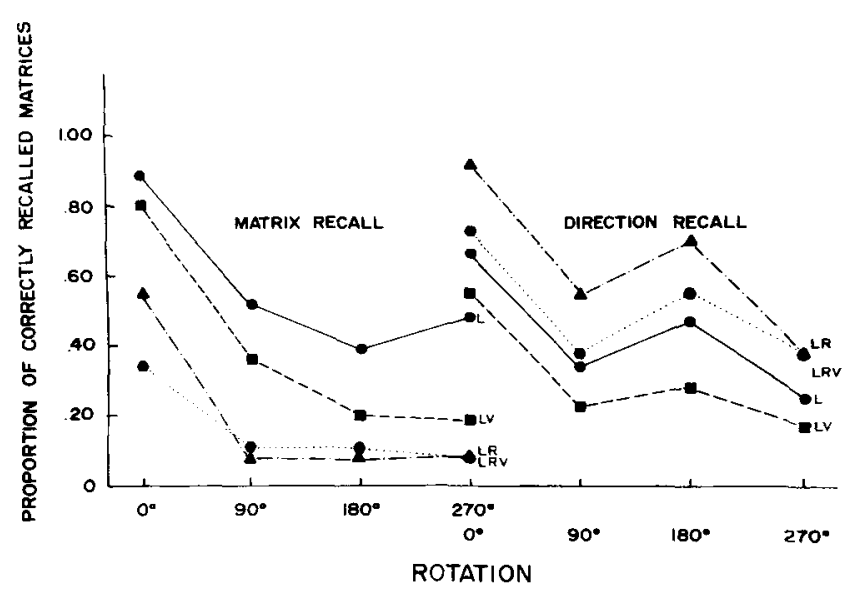

Figure 1. Proportionate matrix and direction recall by Groups L, LV, LR, and LRV, Experiment 1. 
predictions did not posit an interaction between the groups and the recall formats. The last hypothesis was that the characteristics of the input task would interact with the type of recall task.

The data supported the last hypothesis. Under matrix recall, the conditions that fostered formation of mental images, $L$ and $L V$, yielded higher recall than the conditions that required reading which presumably distracted students from imagining the matrices, LR and LRV. However, the two latter conditions produced direction recall that was superior to that shown by Groups $L$ and LV. This interaction was reliable $[\mathrm{F}(3,120)=11.42$, $\mathrm{p}<.01]$.

In general, recall for the direction of movement (47\%) was superior to matrix recall $(32 \%)[\mathrm{F}(1,120)=$ $13.99, \mathrm{p}<.01]$, and the four groups differed $[\mathrm{F}(3,120)=$ $4.03, \mathrm{p}<.01]$. The percentage recall scores for Groups L, LR, LRV, and LV were $50,42,33$, and 32 , respectively. This ordering of the groups did not support predictions that either facilitation or interference would increase as the number of sources of sensory input increased, or that reading would interfere with the construction of an image, thereby reducing recall.

Performance dropped when the matrices were to be rotated $[\mathrm{F}(3,360)=94.27, \mathrm{p}<.01]$. The decline interacted with the recall format $[F(3,360)=7.22, p<.01]$, but not with the groups. The salient feature of this interaction was the direction recall's departure from a continuous decline as the extent of the rotation increased. With direction recall, performance on the 180 -deg rotation was superior to performance on the 90-deg and the 270 -deg rotations. The superiority of $180-\mathrm{deg}$ over other rotations appears to be characteristic of the recall of rotated verbal material (e.g., Kolers \& Perkins, 1975).

Matrix recall dropped from 0-deg to 90-deg rotations and was approximately the same from 90-deg to 270-deg rotations. Figure 1 suggests that all four groups conformed to the general patterns just described for the two recall formats and the failure to find a triple interaction between recall format, rotations, and groups provided statistical corroboration.

The two halves of the experimental trials did not show a reliable main effect $[F(1,120)=1.58, p>.05]$, but the halves interacted with the recall format $[F(1,120)=6.01, p<.05]$. The interaction reflected a practice effect shown by direction recall's improvement from $44 \%$ on the first half of the trials to $50 \%$ on the second half, whereas matrix recall showed no such increase. The percentages for matrix recall on the first and second halves were 32 and 31 , respectively.

\section{Discussion}

The results indicated that (1) the accurate recall of the contents of a 4 by 4 matrix was not systematically related to the number of redundant input modes, thereby refuting hypotheses that predicted simple monotonic relationships between the number of sensory-perceptual input modes and recall, (2) the type of input interacted with the manner of testing recall, although Group $L$ recalled more than the other groups, and (3) the effects of rotating the mental matrix differed as a function of the input-output interaction. These data are compatible with the perspective that the input modes are accorded the processing customarily associated with the sensoryperceptual characteristics of the input mode, that the resultants of this processing (what might be called the "meanings of the messages") may be further processed to form a mental image. Those input tasks which were particularly conducive to the subsequent formation of an image, $L$ and $L V$, yielded higher recall when the demands of the output task were similar to the mental representation. Those input tasks which contained specific works and characteristics also found in the recall test, LR and LRV, were associated with higher recall when the recall test reproduced characteristics of the input task, as did the direction recall task.

\section{EXPERIMENT 2}

Experiment 2 was designed to investigate another dimension of imagery that has not yet been extensively explored: rehearsal effects. We posited that rehearsal opportunities and instructions would facilitate retention if the rehearsal strategy induced a reprocessing or a redintegration of the initial processing accorded the input materials, but would interfere with retention if the rehearsal strategy evoked processes which were distinctly different from the initial processing. The effects upon recall would be the accentuation of the input-output interaction observed in Experiment 1. For example, if the students were told to visualize the imaginary matrix during a rehearsal interval, their performance on matrix recall should be better than if they attempted to verbalize during the rehearsal interval. Students who verbalized during the rehearsal interval following presentation by listening and reading should perform better on direction recall than students who visualized during the rehearsal interval.

Experiment 2 also considered the possibility that, as hypothesized by Brooks (1967), the visual processes induced by the reading requirement of the LR condition interfered with the construction of a mental image. This possibility was tested by introducing a single-input condition for reading $(R)$. If the visual processes associated with reading interfered with the construction of a mental image, it was assumed that matrix recall would be suppressed significantly more than direction recall. Further, the conditions $\mathrm{R}$ and LR should yield approximately the same level of recall which would be lower than that associated with $L$ on matrix recall and higher than $\mathrm{L}$ on direction recall.

To recapitulate, Experiment 2 examined the effects of instructions to visualize the mental matrix, to verbalize the descriptions, or (the controls) of no specific instructions for the rehearsal interval. These rehearsal strategies were crossed with the three input conditions 
of listening ( $\mathrm{L}$ ), listening plus reading (LR), and reading $(\mathrm{R})$, and with the two recall tests, matrix and direction. No rotations were required. The basic hypotheses were that visualizing rehearsal would aid matrix recall more than the verbalizing rehearsal strategy; verbalizing rehearsal would aid direction recall more than visualizing rehearsal. The condition which gave no explicit rehearsal instructions was included as a control to examine the effects of the presence vs. the absence of specific rehearsal instructions. Finally, Experiment 2 introduced rehearsal intervals of two lengths, 0 and $30 \mathrm{sec}$, to examine the likelihood that a certain amount of processing and rehearsal time was necessary for the predicted influences to be manifested.

\section{Method}

Materials. Experiment 2 used the matrices from Experiment 1 , but no rotations were required. The messages were tape recorded. One tape provided a 0 -sec rehearsal interval following the last message for each of the first eight regular matrices and a $30-\mathrm{sec}$ rehearsal interval following the last message for each of the second eight regular matrices; the second tape reversed the procedure so that the messages describing the first eight regular matrices were followed immediately by $30-\mathrm{sec}$ rehearsal in tervals and the messages of the second eight matrices were followed by 0 -sec rehearsal intervals. The tapes always provided a 30 -sec response interval for recording the contents of the matrix on the appropriate recall sheets. Both matrix and direction recall were tested. Finally, the students' perceived use of the rehearsal strategies was assessed with a questionnaire.

Design and subjects. The experimental design consisted of four variables manipulated between subjects and one variable (length of the rehearsal interval, 0 or $30 \mathrm{sec}$ ) within subjects. The four between-subjects variables were the type of recall (matrix or direction), the type of input (listening, L; reading, $\mathrm{R}$; or listening plus reading, LR), the rehearsal instructions (visualizing, verbalizing, none), and the order of blocking the rehearsal intervals $(0-\mathrm{sec}$ before 30 -sec or 30 -sec before 0 -sec). The 288 students were assigned randomly to eight replications of the 2 by 3 by 3 by 2 design. None of the students had participated in Experiment 1.

Procedure. The procedures for the three types of input were similar to those used for Experiment 1, save that Group $R$ read the messages from cards without hearing the tape-recorded messages. The instructions for visualizing rehearsal were: "After the last number has been placed, there will be a blank interval before you are asked to record your responses. During this time, you are to try to focus the matrix clearly in your mind's eye so that you will be able to use this image to make your responses." The instructions for verbalizing rehearsal used the same first sentence and then continued: "During this time, you are to repeat the messages quietly to yourself to help you make your responses." No instructions about the use of the rehearsal interval were given to the subjects assigned to the control condition.

As with Experiment 1, five practice matrices were used to introduce the experimental tasks to the subjects. The 16 regular matrices were presented next, followed by the questionnaire.

\section{Results}

A preliminary analysis indicated that the order of blocking the rehearsal intervals did not produce significant differences. Therefore, the data for the two orders of blocking the lengths of the rehearsal intervals were collapsed in all subsequent analyses.

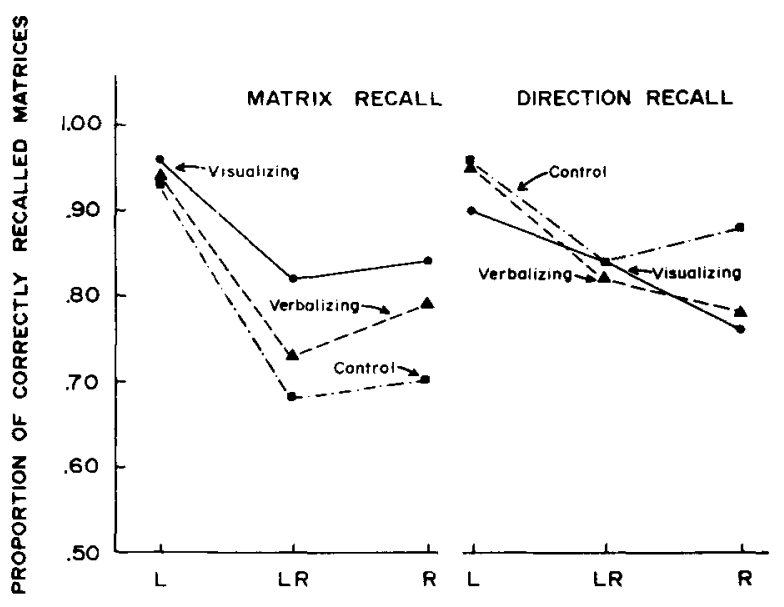

INPUT GROUPS

Figure 2. Proportionate matrix and direction recall by Groups $L, L R$, and $R$ as functions of the rehearsal conditions, Experiment 2.

An analysis of variance was then conducted on the number of matrices recalled correctly as functions of the three between-subjects variables (type of recall test, type of input, rehearsal instructions) and the within-subjects variable (length of the rehearsal interval). This analysis showed that recall differed reliably for the three types of input $[F(2,270)=23.86, p<.01]$. The mean recall scores for Groups L, R, and LR were 7.51, 6.33 , and 6.30 , respectively. Tukey tests showed that the means for Groups R and LR did not differ, but these means were reliably lower than the mean for Group $L$ (Figure 2). These results suggested that reading, whether alone or combined with listening, was less effective as a source of input than listening alone.

The main effect for the type of recall test was not significant $[F(1,270)=3.27, p>.05]$, although the mean direction recall, 6.86 , exceeded the mean matrix recall, 6.57 . The overall superiority by Group L compared to the other groups and the nonsignificant trend for direction recall to exceed matrix recall were similar to the basic results of Experiment 1. However, Experiment 1 also yielded a reliable interaction between the type of input and the type of test that was not found in Experiment 2. In Experiment 2, the interaction between the type of recall test and the type of input was not reliable $[F(2,270)=1.95, p>.05]$. This result contradicted the possibility that visual processes associated with reading would interfere to a greater extent with matrix recall than with direction recall. To determine whether the overall results had masked some significant effects, the individual means contributing to the Type of Input by Type of Test interaction were tested with Tukey tests. No reliable differences emerged, so it seemed reasonable to conclude that the postulated type of interference did not occur. 
The third between-subjects main effect, rehearsal strategy, did not show reliable differences between the mean recall scores for visualizing (6.83), verbalizing (6.67), or control $(6.65)$ conditions $(F<1)$. No main effect for rehearsal strategies had been predicted. However, rehearsal strategies were expected to interact with the recall tasks. When the rehearsal strategy was compatible with the recall task, performance would be enhanced; when the rehearsal strategy was incompatible with the recall task, performance would be impaired. Thus, the visualization rehearsal strategy was expected to induce imaginal processing which would be similar to the matrix recall task and dissimilar from the direction recall task. Hence, matrix recall should exceed direction recall following visualizing rehearsal. Similar reasoning led to the prediction that direction recall should be superior to matrix recall following verbalizing rehearsal. The predicted interaction between rehearsal strategy and type of recall task was reliable $[\mathrm{F}(2,270)=5.01, \mathrm{p}<.01]$. Following visualizing rehearsal, the mean matrix recall was 6.98 and the mean direction recall was 6.68 , whereas following verbalizing rehearsal the mean matrix recall was 6.55 and the mean direction recall was 6.78 . The control condition showed a trend similar to but more exaggerated than the trend for verbalizing rehearsal: The mean direction recall, 7.12 , was considerably higher than the mean matrix recall, 6.17. However, the control condition yielded higher direction recall than either of the two other rehearsal conditions.

Another way of interpreting the above results was to consider the group given no explicit instructions about the rehearsal interval as a control against which to compare the effectiveness of the visualizing and verbalizing rehearsal strategies. From this vantage, both visualizing and verbalizing rehearsal aided matrix recall compared to the control, with visualizing rehearsal producing the greater facilitation. The converse trend was found for direction recall. Both visualizing and verbalizing rehearsal yielded poorer direction recall than the control, with visualizing rehearsal producing the greater loss.

Figure 2 depicts, using proportionate recall, both the interaction between rehearsal strategies and the type of recall test. In addition, Figure 2 shows the overall superiority of listening only. The triple interaction between these three variables was not reliable $(F<1)$.

These results suggest not only that imaginal relationships could be rehearsed, but also that such imaginal rehearsal facilitated compatible recall and hindered incompatible recall. Verbal rehearsal showed parallel effects for direction recall, a type of test that was compatible with verbal rehearsal.

The length of the rehearsal interval was varied in Experiment 2 to determine the length of interval necessary for the rehearsal instructions to be effective. The length of the rehearsal interval did not show a reliable interaction with the rehearsal strategies $[F(2,270)=$ $1.09, \mathrm{p}>.05]$, but did vary with the type of input
$[F(2,270)=3.06, p<.05]$, primarily because recall increased as the length of the rehearsal interval increased for Groups $L$ and LR but showed the opposite trend for Group R. The mean recall scores for Group Lincreased from 7.48 to 7.54 over the rehearsal interval and the scores for Group LR increased from 6.10 to 6.49. The mean recall for Group $\mathbf{R}$, however, declined from 6.41 to 6.26 as the rehearsal interval was increased from 0 to $30 \mathrm{sec}$. No reason for Group R's unexpected reversal was apparent.

\section{Discussion}

Listening only yielded the highest recall regardless of the characteristics of the recall task or the rehearsal strategies. Experiment 2, however, did not evince the interaction between type of input and the recall test found in Experiment 1. These differences probably arose from the different use of input time for rehearsal by the three input groups. Some students in Group L spontaneously remarked that they had been rehearsing as they listened to the messages. Apparently, listening only provided more rehearsal opportunities during input than the other two input conditions.

The effects of the various types of rehearsal instructions differed for the two recall tasks in ways which were predictable from the characteristics assumed to be instituted by the rehearsal instructions and the salient aspects of the recall tasks. Visualizing rehearsal facilitated matrix recall more than the other rehearsal strategies. Verbalizing rehearsal aided direction recall more than did visualizing rehearsal, but the control rehearsal condition yielded the highest direction recall. These results were interpreted as indicating that images can be rehearsed and that this rehearsal may be beneficial if the processes being instituted are compatible with the processes instituted by the recall tests. Conversely, when the processes reinstituted by rehearsal were incompatible with the processes induced by the recall test, poorer recall should have been observed. And, as noted above, explicit rehearsal instructions tended to interfere with direction recall compared to performance following no explicit rehearsal instructions.

Finally, direction recall by Group $\mathrm{R}$ was not reliably higher than matrix recall by Group $R$, was would have been expected if the visual processes associated with reading interfered with visualizing and with subsequent matrix recall.

\section{GENERAL DISCUSSION}

Our results indicated that an adequate theory of imagery must encompass the conditions under which rehearsal of images will facilitate or hinder recall, the kinds of input effects demonstrated herein modified by the demands of the output task, and the differences in the recall of rotated images as functions of both the input characteristics and the extent of the rotation. One model 
that enjoyed some empirical support was a modified levels of processing approach.

This model posited that the input messages would undergo the structural and phonemic processing characteristically accorded the type of input. Additional semantic processing would result in what might be termed the "meaning" of the input messages. Subsequent processing could occur, if dictated by the experimental tasks. Thus, when subjects were instructed to construct a mental image, the products of earlier processing would undergo whatever additional, elaborative processing is identified as the "construction of a mental representation." This model is, of course, a slight modification of the approach proposed by Craik and his colleagues (Craik \& Lockhart, 1972; Lockhart et al., 1976).

We proposed, as they have done, that the processing by-products sustain memory so that the more elaboratively processed materials were likely to be recalled more often than the shallowly processed materials, and that rehearsal which reinstituted many aspects of the original processing also should enhance recall. Recall itself would be influenced by the extent to which the retrieval instututed processes similar to those associated with initial or rehearsal processing.

The above model predicted superior recall when similar processing was induced by the input or the rehearsal strategy and the characteristics of the recall test, that is, when these processes were compatible. When differing processes were induced during encoding, rehearsal, or retrieval, recall would suffer. The predicted enhancement of recall occurred in Experiment 1 when the input characteristics (Groups L and LV) presumably fostered imagery that was tested with matrix recall and when the input characteristics (Groups LR and LRV) encouraged verbal encoding that was probed by direction recall. The rehearsal results of Experiment 2 also provided support. Visualizing rehearsal produced higher matrix recall than the other rehearsal conditions; the control rehearsal condition and verbalizing rehearsal yielded higher direction recall than the visualizing rehearsal condition. Presumably, the self-instructed rehearsal strategies that occurred in the control condition of Experiment 2 yielded processing that was more compatible with the encoding and retrieval processes associated with reading input and direction recall than the other rehearsal strategies. Thus, both experiments implicated the compatibility, similarity, or overlap between the likely encoding and retrieval processes as the salient characteristics in the imagery paradigm.

It must be noted, however, that Group $L$ recalled more matrices correctly, in both experiments, than any of the other input groups. This result appeared to conflict with the view that the effective determinant of performance was the compatibility or overlap between the encoding and retrieval processes. In Experiment 1, of course, the input conditions interacted with output conditions so that Group L's recall was superior to that of all the other groups for matrix recall but not for direction recall. In Experiment 2, Group $L$ showed superior matrix and direction recall, regardless of the rehearsal conditions to which they had been assigned. The most reasonable explanation of the superiority of Group $L$ on direction recall of Experiment 2 was that Group L subjects began rehearsal earlier than subjects assigned to the other groups. Students' postexperimental comments supported this view. Experiment 1 did not have a rehearsal interval and presumably did not encourage rehearsal to the same extent as Experiment 2.

Further, the rotation of the information, encoded as either a visualized or a verbalized representation, was consonant with the standard findings that verbal materials would be recalled better at a $180-\mathrm{deg}$ rotation than at either $90-\mathrm{deg}$ or $270-\mathrm{deg}$ rotations, whereas the recall of imaginal material declined as the extent of the rotation increased. These results are interesting because they indicate that the imagery paradigm yields evidence which is similar to that found with other materials and techniques.

What do the results portend for the levels of processing position? We believe that they argue forcefully that recall is facilitated by a reinstitution or reconstruction of the processing accorded the information. Further, the data from Experiment 2 suggest that recall was aided more by reconstruction of the rehearsal processing than by reconstruction of the initial input processing. Thus, these results implicate a type of encoding specificity which provides for rehearsal-produced modification of initial encoding. The greater effectiveness of rehearsal than input processing may operate in various ways. For example, rehearsal processing may obliterate initial processing; it may override initial processing by virtue of being more recent and/or more salient; it may combine the similar types of processing associated with initial encoding and later rehearsal of like characteristics.

The extant results would be difficult to explain in terms of the depth of processing; for example, when the same basic message was received by the input groups. The evidence was more supportive of an elaboration of processing position. The elaboration may occur in numerous ways, including both imagery and verbal types of processing. The data did not indicate the imagery provided greater elaboration than verbal processing.

While the model outlined above appeared to be particularly appealing, the predictions extracted for the experiments reported herein were virtually indistinguishable from predictions which could be generated from more associative models such as those of Anderson (1976) and Paivio (1976). Anderson hypothesized that imagery is the result of elaborative productions (propositions). That is, through experience, productions are acquired which may be used to "enrich" or further elaborate a pattern. Paivio postulated that two major types of representations or systems may be used (imagi- 
nal and verbal) to encode, store, organize, and retrieve material. These two types of representations operate independently but are partially interconnected. The notions of levels of processing and output compatibility have been considered in this theory, as Paivio (1976) noted.

Whatever model is used, it must accommodate the findings that the imagery-encouraging input ( $L$ and $L V$ ) and the visualizing rehearsal strategies enhanced matrix recall more than other rehearsal strategies or input conditions and that the verbal-preserving input (LR and LRV), the control, and the verbalizing rehearsal strategy enhanced direction recall more than the visualizing rehearsal.

\section{REFERENCES}

ANDERSON, J. R. Language, memory, and thought. Hillsdale, N. J: Erlbaum, 1976.

Brooks, L. R. The suppression of visualization by reading. The Quarterly Joumal of Experimental Psychology, 1967, 19, 289-299.

CRAIK, F. I. M., \& Lockhart, R. S. Levels of processing: A framework for memory research. Joumal of Verbal Leaming and Verbal Behavior, 1972, 11, 671-684.

COOPER, L. A. Mental rotation of random two-dimensional shapes. Cognitive Psychology, 1975, 7, 20-43.
Cooper, L. A., \& Saepard, R. N. Chronometric studies of the rotation of mental images. In W. G. Chase (Ed.), Visual information processing. New York: Academic Press, 1973.

KoleRs, P. A. The recognition of geometrically transformed text. Perception \& Psychophysics, 1968, 3, 57-64.

Kolers, P. A., \& Perurins, D. N. Orientation of letters and errors in their recognition. Perception \& Psychophysics, 1969, 5, 265-269.

Kolers, P. A., \& Perkins, D. N. Spatial and ordinal components of form perception and literacy. Cognitive Psychology, 1975 , 7, 228-267.

Lockrart, R. S., Craik, F. I. M., \& JaCoby, L. Depth of processing, recognition and recall. In J. Brown (Ed.), Recall and recognition. London: Wiley, 1976.

PAIvio, A. Imagery in recall and recognition. In J. Brown (Ed.), Recall and recognition. London: Wiley, 1976.

PosNer, M. I., \& SNYDER, C. R. R. Attention and cognitive control. In R. L. Solso (Ed.), Information processing and cognition: The Loyola symposium. Hillsdale, N. J: Erlbaum, 1975.

Smith, K. U., Cambria, R., \& Steffan, J. Sensory-feedback analysis of reading. Journal of Applied Psychology, 1964, 48. 275-286.

WICKELGREN, W. A. The long and the short of memory. In D. Deutsch and J. A. Deutsch (Eds.), Short-term memory. New York: Academic Press, 1975.

(Received for publication December 12, 1976; revision accepted March 11.1977.) 